\title{
A BENEFICIAL ANALYSIS OF NODE DEPLOYMENT SCHEMES FOR WIRELESS SENSOR NETWORKS
}

\author{
G Sanjiv Rao ${ }^{1}$ and V Vallikumari ${ }^{2}$ \\ ${ }^{1}$ Associate Professor, Dept of IT, Sri Sai Aditya Institute of Science \\ And Technology, Surampalem, AP India. \\ sanjiv_gsr@yahoo.com \\ ${ }^{2}$ Professor, Dept of CS\&SE, College of Engineering, Andhra University \\ Visakhapatnam, AP, India. \\ vallikumari@gmail.com
}

\begin{abstract}
The Recent advances in electronics and wireless communication technologies have enabled the development of large-scale wireless sensor networks that consist of many low-powers, low-cost and smallsize sensor nodes. Sensor networks hold the promise of facilitating large-scale and real time data processing in complex environments. Some of the application areas are health, military, and home. In military, for example, the rapid deployment, self-organization, and fault tolerance characteristics of sensor networks make them a very promising technique, for military command, control, communications, computing, and the targeting systems. Deployment of nodes in Wireless Sensor Networks (WSNs) is a basic issue to be addressed as it can influence the performance metrics of WSNs connectivity, resilience and storage requirements. Many deployment schemes have been proposed for wireless sensor networks. In this paper we consider the implications of various deployment schemes for the connectivity and resilience of the WSNs. Our approach to deal with the affective trade-offs between resilience, connectivity and storage requirements for each deployment schemes in the literature, we survey four deployment models random, grid, group and grid-group to show which deployment scheme can be used to increase network connectivity, without increasing storage requirements or sacrificing resilience with respect to some factor. Our contribution is we had implemented of WSNs using random, grid and grid-group deployment Knowledge. WSNs have been simulated with Network Simulator 2.34 for node configuration, sink node configuration, topology creation, with sensing capabilities, temperature and energy by using Mannasim
\end{abstract}

\section{KEYWORDS}

Wireless Sensor Network, Grid deployment, Random deployment, Grid- Group deployment,

\section{INTRODUCTION}

A wireless sensor network (WSN) is a network formed by a large number of sensor nodes, each equipped with sensors to detect physical phenomena such as heat, light, motion, or sound. Using different sensors, WSNs can be implemented to support many applications including security, entertainment, automation, industrial monitoring, public utilities, and asset management. However, many WSN devices have severe resource constraints in terms of energy, computation, and memory, caused by a need to limit the cost of the large number of devices required for many applications and by deployment scenarios that prevent easy access to the devices. Such resource limitations lead to many open issues. In a sensor network, many tiny computing nodes called

DOI: $10.5121 /$ ijassn.2012.2204 
sensors are scattered in an area for the purpose of sensing some data and transmitting data to nearby base stations for further processing. A sensor node, also known as a mote, is a node in a wireless sensor network that is capable of performing some processing, gathering sensory information and communicating with other connected nodes in the network. The transmission between the sensors is done by short range radio communications. The base station is assumed to be computationally well-equipped whereas the sensor nodes are resource- starved. The sensor nodes are usually scattered in a sensor field (i.e., deployment area or target field) as shown in Figure 1 each of these scattered sensor nodes has the capabilities to collect data and route data back to the base station. Data are routed back to the base station by a multi-hop infrastructure-less architecture through sensor nodes. The base station may communicate with the task manager node via Internet or Satellite An adhoc network is a group of mobile, wireless hosts which cooperatively and spontaneously form a network independently of any fixed infrastructure or centralized administration. In particular, an adhoc network has no base stations, host, also called node, communicates directly with nodes within its wireless range and indirectly with all other destinations using a multi-hop route through other nodes in the network [1].

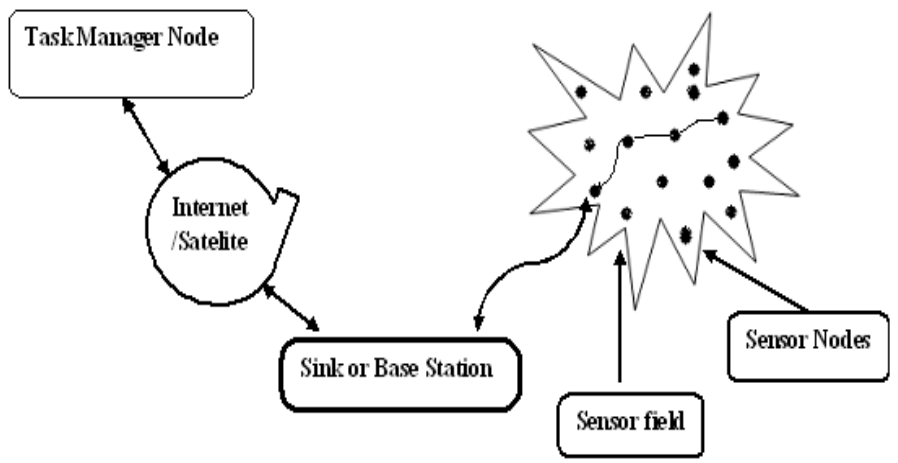

Figure 1. Sensor nodes scattered in a target field

Many applications require WSNs to exchange sensitive information or contain feedback processes that have high reliability requirements, and they require a high level of security to succeed. Yet, strong security is difficult to achieve with resource-limited sensor nodes, and many well-known approaches become infeasible. In this paper, we explore the node deployment models in WSNs. A WSN can be composed of homogeneous or heterogeneous sensors, which possess the same or different communication and computation capabilities, respectively. However we consider the homogeneous WSNs which can provide less complexity and better manageability.

WSN composed of ten sensor nodes deployed randomly around a base station. Depending on the size of the deployment area, the transmission range of the sensor nodes, and the base station, sensor nodes can communicate with the base station directly or indirectly by computing a hop-byhop route to it [2].In WSNs, two specific factors arise: (1) the envisioned applications and the operation of the protocol layers are usually driven by the physical variables measured by the sensors. Therefore, the dynamics of the physical parameters sensed by the network govern the network traffic, and even the topology. (2) The energy is a primary concern in WSN. Usually, nodes run on non-rechargeable batteries. Therefore, the expected node lifetime is a fundamental element that must be taken into account. Recent technological advances have made it possible to have various deployment strategies for wireless sensor networks consisting of a large number of low-cost, low power, and multifunctional sensor nodes that communicate at short distances through wireless links. In our study, we illustrate various node deployment strategies for wireless sensor networks: a random, a grid, a group and a grid-group. We analyze three performance metrics: connectivity, resilience and low memory usage. 


\section{DEPLOYMENT SCHEMES FOR WSNS}

In WSNs, the major challenge is the deployment of the nodes in the deployment region to satisfy continuous sensing with extended network lifetime while maintaining uniform coverage. Various architectures and node deployment strategies have been developed for wireless sensor network, depending upon the requirement of application [3] we focus on five deployment schemes for sensor networks environments, random deployment, grid deployment, group-based deployment, and grid-group deployment.

\subsection{Random Deployment}

Random deployment means setting positions of wireless sensor nodes randomly and independently in the target area. On the other hand, in precise deployment, nodes are set at exact positions one by one according to the communication range of the nodes. Usually, the positions are chosen to minimize the number of nodes required to achieve certain deployment goal. However, precise deployment method is time consuming even though costing the least number of nodes. Random deployment method is fast in practice though costs a relatively larger number of nodes to achieve the same deployment goal. When practical application scenarios are considered, random deployment is a feasible and practical method, and sometimes it is the only feasible strategy [4]. Random approach for node deployment is deeply discussed in [5], which has considered as one of the competitors.

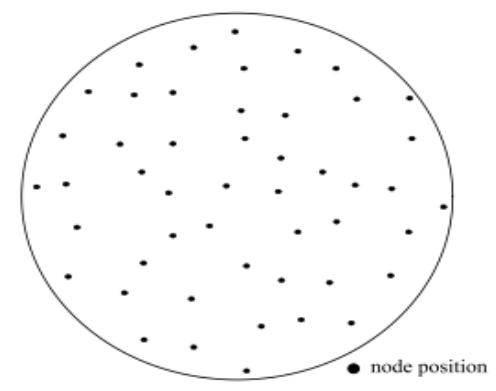

Figure 2. Random Deployment

In this deployment, as shown in Figure 2 each of the $\mathrm{n}$ sensors has equal probability of being placed at any point inside a given target field. Consequently, the nodes are scattered on locations which are not known with certainty. For example, such a deployment can result from throwing sensor nodes from an airplane. In general, a uniform random deployment is assumed to be easy as well as cost-effective.

\subsection{Grid Deployment}

So far, we have only considered the non-deterministic deployment scheme, random deployment, in which the sensor nodes are thrown randomly to form a network. However, since excess redundancy is required to overcome uncertainty it could be very expensive. In [3], it has state that grid deployment is an attractive approach for moderate to large-scale coverage-oriented deployment due to its simplicity and scalability. In a grid deployment the amount of connectivity and resilience of the sensor nodes against the adversaries when they are deployed in grid fashion. Grid deployment is conducted by dropping sensors row-by row using a moving carrier. Previously many studies have explored the properties of grid deployment in the ideal circumstance where individual sensors are placed exactly at grid points. However, in practice, it is 
often infeasible to guarantee exact placement due to various errors, including misalignments and random misplacement.

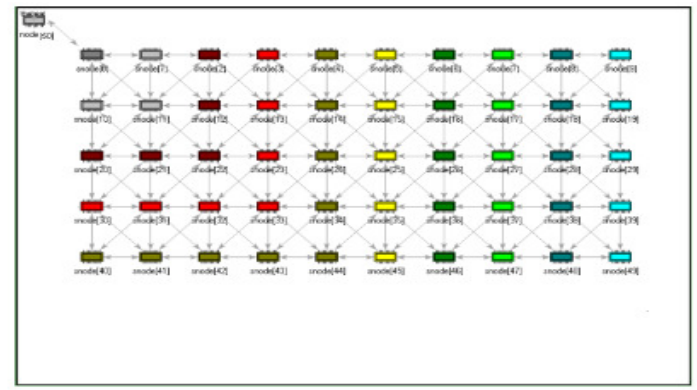

Figure 3. Grid Deployment

There are three popular grid layouts namely, unit square, an equilateral triangle, a hexagon, etc. Among them, we investigate a square grid because of its natural placement strategy over a unit square. A grid deployment is considered as a good deployment in WSN, especially for the coverage performance. Figure 3 shows a grid deployment of sensors for a wireless sensor network. The desired distance between consecutive droppings is achieved by controlling the time intervals. However, due to placement errors, this ideal deployment is not realistic. In the unreliable sensor grid model, $\mathrm{n}$ nodes are placed on a square grid within a unit area, with a certain probability that a node is active, and a defined transmission range of each node [3].It is a challenging issue to add nodes to ensure wireless connectivity, particularly when there are location constraints in a given environment that dictate where nodes can or cannot be placed. If the number of available nodes is small with respect to the size of the operational area and required coverage, a balance between sensing and routing nodes has to be optimized. Hence we can say that random deployment is more energy efficient than grid deployment.

\subsection{Group based Deployment}

We have considered the networks in which sensors are deployed in groups such that sensors from a group are closer together on average than sensors from different groups. They refer to this as group deployment of sensors. we assumed that group deployment may be used in order to improve the coverage of the target region by sensors, as it provides more control over the physical distribution of sensors, and also a convenient way of carrying out the deployment: in the case where several vehicles are available for distributing sensors, they could be used to deliver sensors to different portions of the target area simultaneously. However, the main motivation for considering group deployment from the point of view of key distribution is the fact that the partial location knowledge it provides can be used in order to improve the connectivity of the network.

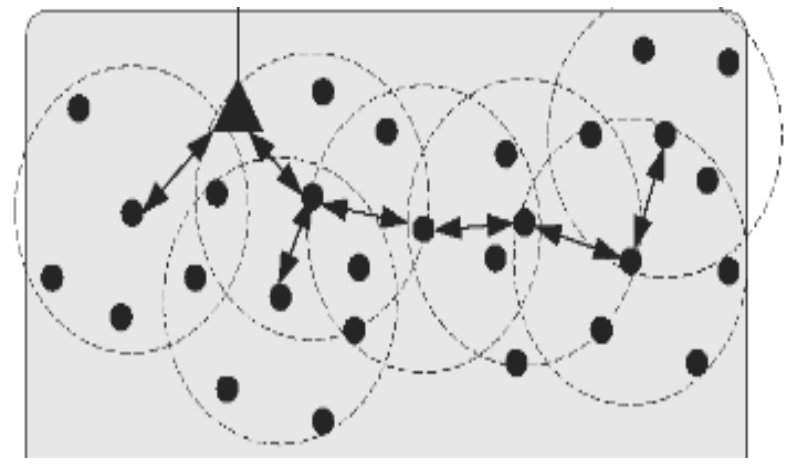

Figure 4. Group based deployment 
In a group-based deployment model [6] to improve key predistribution. In this model, it states that sensor nodes are only required to be deployed in groups. The critical observation that the sensor nodes in the same group are usually close to each other after deployment. This deployment model is practical, it greatly simplifies the deployment of sensor nodes, while still providing an opportunity to improve key predistribution. As Figure 4 shows a large-scale distributed sensor network composed of a large number of low-power sensor devices. Typically, these networks are installed to collect sensed data from sensors deployed in a large area. Sensor networks often have one or more centralized controllers called base stations or sinks. A base station, usually many orders of magnitude more powerful than a sensor, is typically a gateway to other networks or data centers via high bandwidth communication links. In this scheme, a sensor deployment area is first partitioned into multiple small square areas called zones and then sensors deployed in each zone form a group. This design can restrict the consequence of attacks within a small range.

\subsection{Grid- Group Deployment}

Grid-group deployment scheme for wireless sensor networks is discussed in [7], when nodes are deployed in a region, all nodes need not communicate with all other nodes in the network. Due to limited power, all nodes cannot communicate with all other nodes. So we divide the entire region into equal-sized squares or grids as done in Liu and Ning [2003, 2005], and Huang and Medhi [2007]. In this scheme has the advantage that all nodes within a particular region can communicate with each other directly and nodes which lie in a different region can communicate via special nodes called agents which have more resources than the general nodes as shown in Figure 5 It is also assumed that it is more difficult to compromise an agent than a sensor node. Whatever the size of the network, the number of agents in a region is always three. This scheme ensures that even if one region is totally disconnected, the regions are not affected.

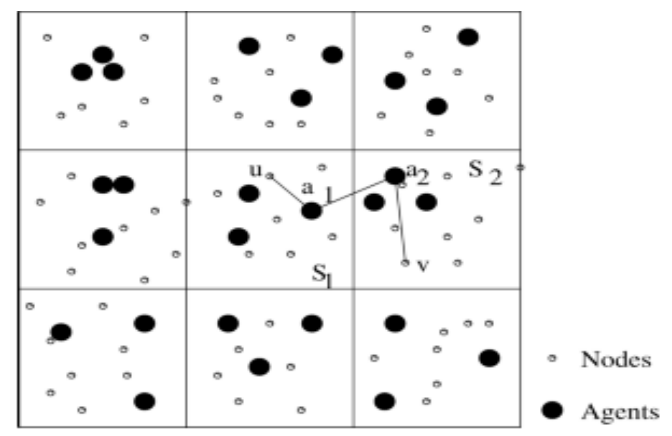

Figure 5. Grid group deployment

In this we assumed that grid-group deployment provides a very good resiliency in terms of the fraction of nodes disconnected and regions disconnected.

\section{RELATED WORK}

In this paper WSNs has been simulated with Network Simulator 2.34 for node configuration, sink node configuration, topology creation, and sensor node has been deployment in random, grid and grid-group models, and sensor node has been configured with sensing, temperature, energy capabilities by using Mannasim. 


\subsection{Simulation with NS-2.34 and Mannasim}

NS-2.34 is a discrete event network simulator that has developed in 1989 as a variant of the REAL network simulator. Initially intended for wired networks, the Monarch Group at CMU has extended NS-2 to support wireless networking. NS-2's code source is split between C++ for its core engine and $\mathrm{OTcl}$, an object oriented version of TCL for configuration and simulation scripts. The combination of the two languages offers an interesting compromise between performance and ease of use. Implementation and simulation under NS-2 consists of 4 steps (1) Implementing the protocol by adding a combination of C++ and OTcl code to NS-2's source base (2) Describing the simulation in an OTcl script (3) Running the simulation and (4) Analyzing the generated trace files.NS-2 is powerful for simulating ad-hoc networks. But to simulate WSN in NS-2, it needs to have additional module to represent the protocols specific to WSN.

MANNASIM is a framework for WSN simulation based on NS-2. It extends NS-2 by introducing new modules for design, development and analysis of different WSN applications. The goal of MANNASIM is to develop a detailed simulation framework, which can accurately model different sensor nodes and applications while providing a versatile test bed for algorithms and protocols The MANNASIM Framework is a module for WSN simulation for development and analysis of different WSN applications.

\subsection{Node Configuration}

Node-configuration for a wireless, mobile node that runs AODV as its adhoc routing protocol in a hierarchical topology would be as shown below. We decide to turn tracing on at the agent and router level only. Also we assume a topology has been instantiated with "set topo [new Topography]".

\section{Example of a node-config command}

\$ns_node-config -addressType hierarchical

-adhocRouting AODV

-llType LL

-macType Mac/802_11

-ifqType Queue/DropTail/PriQueue

-ifqLen 50

-antType Antenna/OmniAntenna

-propType Propagation/TwoRayGround

-phyType Phy/WirelessPhy

-topologyInstance \$topo

-channel Channel/WirelessChannel

-agentTrace ON

-routerTrace ON

-macTrace OFF

-movementTrace OFF

-propType Propagation/TwoRayGround

\subsection{Sensor Node Configuration}

Sensor node can be configured so that an Antenna and physical layer configurations should be set.

Example of an Antenna Setting

Antenna/OmniAntenna set X_0 
Antenna/OmniAntenna set $\mathrm{Y}_{-} 0$

Antenna/OmniAntenna set $Z_{-} 1.5$

Antenna/OmniAntenna set Gt_ 1.0

Antenna/OmniAntenna set $\mathrm{Gr}_{-} 1.0$

\section{Example of a Sensor node -config setting}

\$ns_node-config -sensorNode ON

-adhocRouting \$val(rp)

-adhocRouting \$val(rp)

-llType \$val(1l)

-macType \$val(mac)

-ifqType \$val(ifq)

-ifqLen \$val(ifqlen)

-antType \$val(ant)

-propType \$val(prop)

-energyModel \$val(en)

Sensor node can be configured so that a specified amount of energy will be deducted from its energy reserve each time it receives a phenomenon broadcast. To set this up, include the following parameters in the sensor node's node-config routine.

\section{Example of sensing capabilities}

Node/MobileNode/SensorNode set sensingPower_ 0.015

Node/MobileNode/SensorNode set processingPower_ 0.024

Node/MobileNode/SensorNode set instructionsPerSecond_ 8000000

\subsection{Simulation of Random Deployment}

Random deployment means setting positions of wireless sensor nodes randomly and independently in the target area. On the other hand, in precise deployment, nodes are set at exact positions one by one according to the communication range of the nodes. In Figure 5 we visualization of a simulated sensor network with 10 nodes deployed with random deployment scheme.

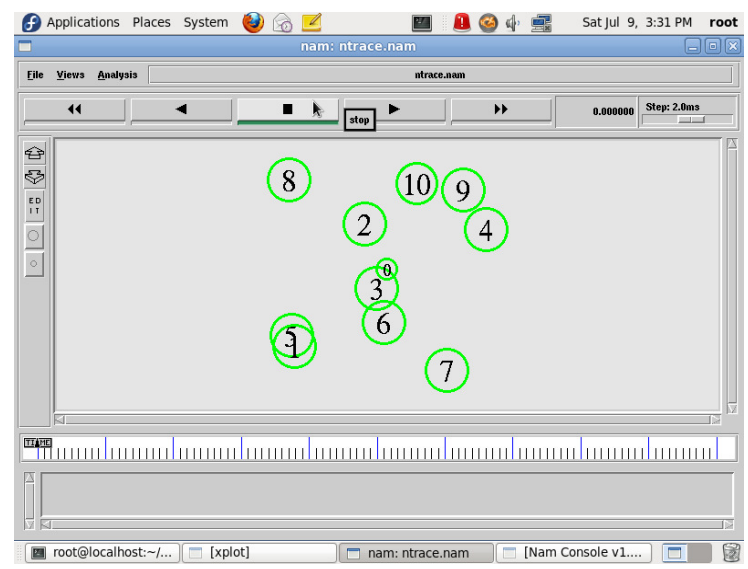

Figure 6. Simulation output of Random Deployment 


\subsection{Simulation of Grid Deployment}

Figure 7 shows the visualization of a simulated sensor network with 10 nodes deployed with grid deployment scheme, and grid deployment is conducted by dropping sensors row-by row using a moving carrier in the target field.

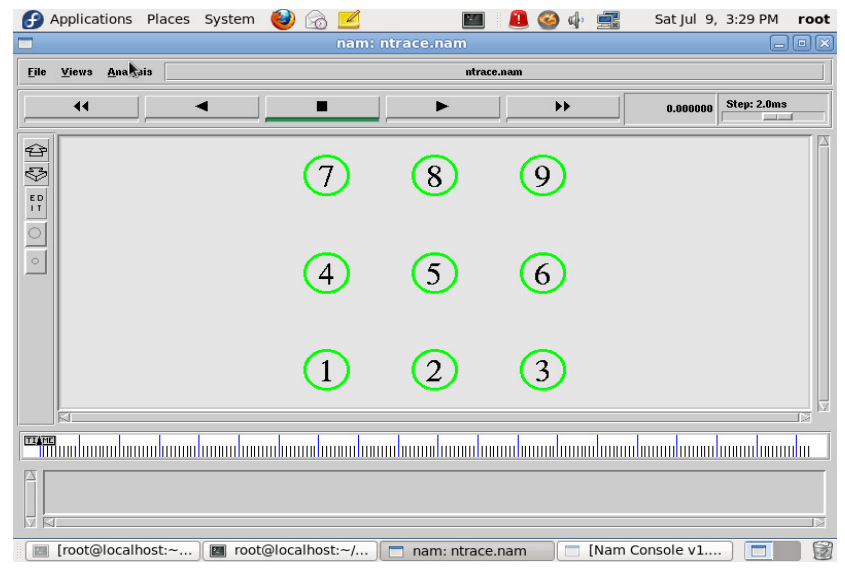

Figure 7. Simulation output of Grid deployment

\section{Example Grid deployment procedure}

proc get_next_x \{\}

\{ global val

set aux [expr int(sqrt(\$val(nn)))]

return [expr ((\$node )\% \$aux)* \$val(x) / (\$aux - 1)] \}

proc get_next_y \{\}\{

global val

set aux [expr int(sqrt(\$val(nn)))]

return [expr ((\$node ) / \$aux $) *$ \$val $(\mathrm{y}) /($ aux -1$)] \quad\}$

Figure 8 shows that wireless sensor network has simulated and sensor nodes have been deployed by grid deployment scheme, hence the sensing capabilities, propagation, processing capability, data generator, setting has configured.

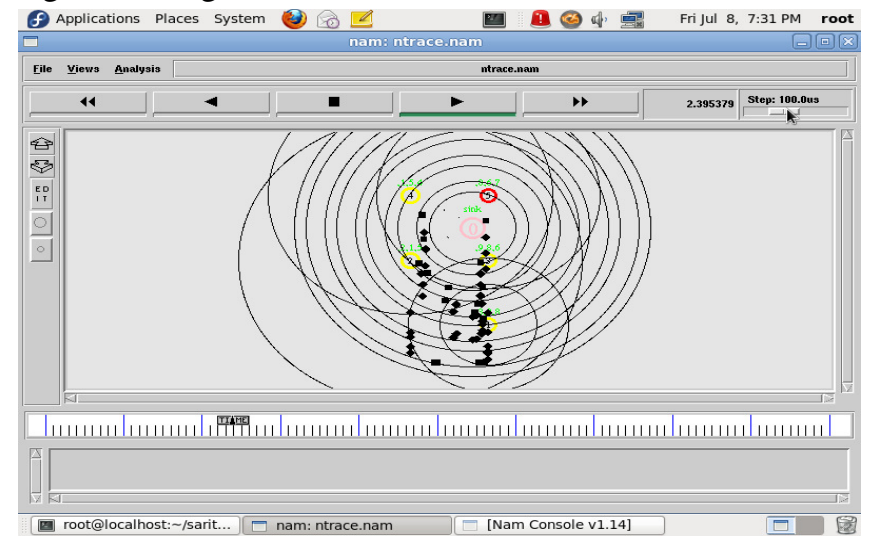

Figure 8. Data transfer and sensing capabilities for WSNs 


\subsection{Simulation of Grid- Group Deployment}

Figure 9 shows the visualization of a simulated sensor network with 10 nodes deployed with each grid- group deployment scheme, in this figure there are three sink nodes $0,11,22$ and each sink node consists 10 nodes deployed in a grid manner by dropping sensors row-by row using a moving carrier in the target field.

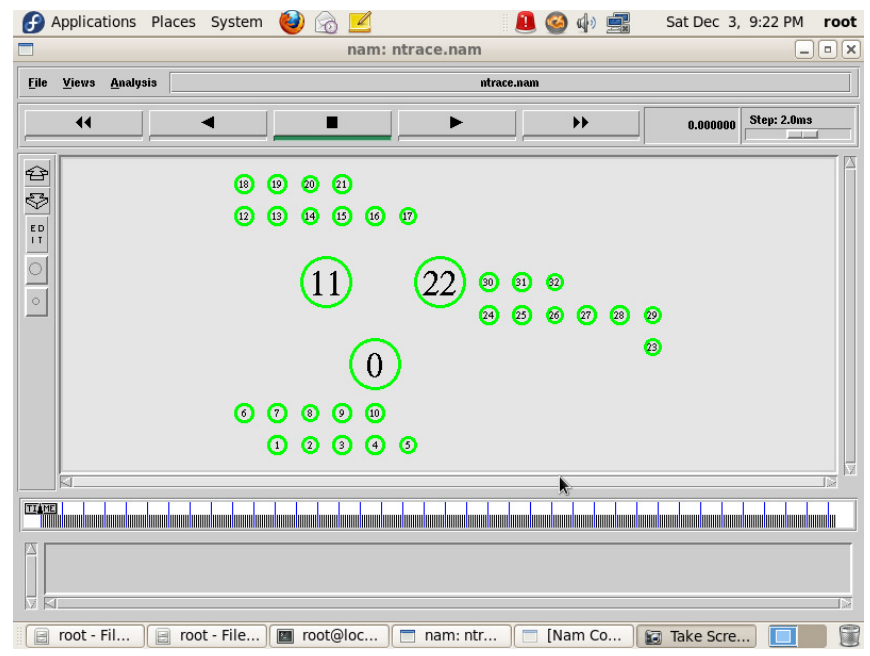

Figure 9. Simulation output of Grid-Group Deployment

Figure 10 shows that wireless sensor network has simulated and sensor nodes have been deployed by grid-group deployment scheme, hence all the node can communicate each other in between the grid-group, which consists of the sensing capabilities, propagation, processing capability, data generator, setting has configured.

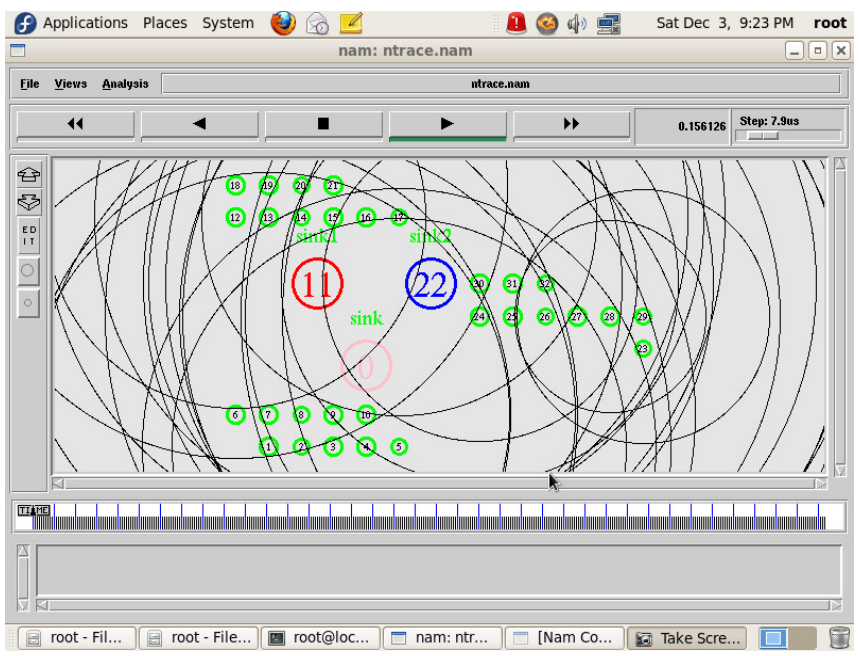

Figure 10. Sensing capabilities for Grid-Group 


\subsection{Comparison with performance metrics: Connectivity, Storage, Scalability}

Thus, so far we have only considered various node deployment schemes for wireless sensor networks. In this we describe the comparative study of these node deployment schemes.

Table 1. Comparison of the Different Schemes with Connectivity, Storage and Scalability.

\begin{tabular}{|l|l|l|l|l|}
\hline Deployment & Nodes & Connectivity & Storage & Scalability \\
\hline Random & Homogenous & Very poor & $\tau(\lambda+1)$ & Scalable \\
\hline Grid & Homogenous & Poor & $\begin{array}{l}\mathrm{O}(\sqrt{\mathrm{N}}) \\
.\end{array}$ & Not Scalable \\
\hline Grid- Group & Homogenous & Very good & $\begin{array}{l}\mathrm{O}(\operatorname{logN}) 1 \\
\mathrm{O}(\operatorname{logN}) 2\end{array}$ & Not Scalable \\
\hline
\end{tabular}

Table 2. Comparative Study of Deployment Schemes with the Resiliency

\begin{tabular}{|l|l|l|}
\hline Deployment & $\begin{array}{l}\text { Random Node } \\
\text { Capture Attack }\end{array}$ & $\begin{array}{l}\text { Selective Node } \\
\text { Capture Attack }\end{array}$ \\
\hline Random & Exists & Exists \\
\hline Grid & Exists & Exists \\
\hline Grid- Group & Exists & Does not exits \\
\hline
\end{tabular}

\section{Conclusions}

In this paper, we focus on various node deployment schemes for wireless sensor networks, and our study is based on a network model in which homogeneous nodes distributed in a target area. We also simulated WSNs with NS-2.34 and Mannasim and sensor nodes has been deployed by random, grid and grid-group deployment scheme and configured the sensing parameters and also presented the comparative study of those schemes with respect to various performance metrics: connectivity, storage, and resilience. It can be extended for WSNs security requirements, for secure data transmission.

\section{ACKNOWLEDGEMENTS}

The authors would like to thank to College of Engineering, Andhra University, Visakhapatnam and Sri Sai Aditya Institute of Science and Technology, Surampalem authorities for their constant support and cooperation!

\section{REFERENCES}

[1] Ian F. Akyildiz, Weilian Su, Yogesh Sankarasubramaniam A Survey on Sensor Networks IEEE Communications Magazine • August 2002.

[2] WANG, DU, and LIU 2009. ShortPK: A Short- Term Public Key Scheme for Broadcast Authentication in Sensor Networks. ACM Transactions on Sensor Networks, Vol. 6, No. 1, Article 9

[3] Messai, Aliouat, and Seba. 2010. Tree Based Protocol for Key Management in Wireless Sensor Networks. Research Article, EURASIP Journal on Wireless Communications and Networking. 
[4] Monica and Ajay K Sharma. Comparative Study of Energy Consumption for Wireless Networks based on Random and Grid Deployment Strategies. International Journal of Computer Applications (0975 - 8887), volume 6-no.1, September 2010.

[5] XiangYang, Yajun Wang, Wangsen Feng, Mo Li, and YunHao Liu. Random Deployment of wireless sensor Networks: Power of Second chance .

[6] Wint Yi Poe, and Jens B. Schmitt. Node Deployment in Large Wireless Sensor Networks: Coverage, Energy Consumption, and Worst-Case Delay. AINTEC'09, November 18-20, 2009, Bangkok, Thailand)

[7] Liu, D., Ning, P., and Du, W. 2008. Group-based key predistribution for wireless sensor networks. ACM Trans. Sens. Netw. 4, 2, Article 11 (March 2008)

\section{Authors}

G.Sanjiv Rao is currently working as Associate Professor in IT Department, Sri Sai Aditya Institute of Science \& Technology Surampalem, AP, India. He is working towards Ph.D. at College of Engineering, Andhra University. He received is M.Tech from the same institute. His research interests are in the areas of Security over Wireless Sensor Networks.

Dr. V .Valli kumari is currently Professor in Department of Computer and Systems Engineering, College of Engineering, Andhra University Visakhapatnam, AP,India . She received her Ph.D. from Dept of CS\&SE, College of Engineering Andhra University. Her research interests include Security and Privacy issues in Data Engineering, Network Security and E-Commerce. She is a member of IEEE and ACM and fellow of IETE.
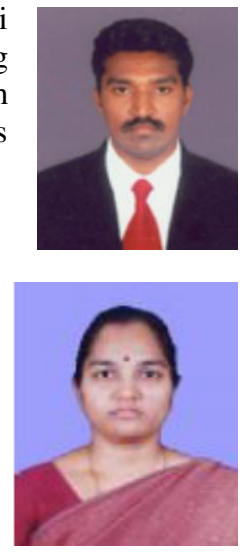